\title{
Uso de dispositivos eletrônicos para avaliar o comportamento de pastejo de bovinos
}

\section{Use of electronic devices to evaluate cattle grazing behavior}

\author{
Natalia Maria Alejandra Aguilar $r^{1,2,3^{*}}$, Franciely de Olivera Costa 3,4 \\ ${ }^{1}$ Instituto Nacional de Tecnología Agropecuaria (INTA), Colonia Benítez, Chaco, Argentina \\ ${ }^{2}$ Facultad de Ciencias Veterinarias, Universidad Nacional del Nordeste (UNNE), Corrientes, Argentina \\ ${ }^{3}$ Grupo de Estudos e Pesquisas em Etologia e Ecologia Animal (ETCO), Universidade Estadual Paulista (Unesp) Jaboticabal, SP, Brasil \\ ${ }^{4}$ Universidade Estadual Paulista (Unesp), Jaboticabal, SP, Brasil
}

\section{Resumo}

O estudo do comportamento de pastejo em bovinos pode ser considerado uma ferramenta importante para entender porque os animais escolhem algumas plantas ou momentos para pastejar. Para estudar o comportamento de pastejo, geralmente é desejável que as observações sejam feitas ao longo das 24 horas do dia. Observações deste tipo, no entanto, não são fáceis de realizar quando dependem da presença de observadores humanos, devido a uma série de dificuldades na coleta de dados e erros de registro ou imprecisão na identificação da área de estudo. Nos estudos de comportamento alimentar, em ungulados selvagens e domésticos, o uso de tecnologias de precisão procura facilitar a coleta de informações sobre os animais e o ambiente, reduzindo a interferência da presença dos observadores sobre o comportamento dos mesmos. 0 objetivo desta revisão foi identificar alguns dispositivos eletrônicos como possíveis ferramentas para avaliar o comportamento de pastejo de bovinos de corte em sistemas extensivos. Os primeiros dispositivos desenvolvidos apresentaram algumas limitações para o seu uso em grandes áreas ou para registrar e armazenar informações comportamentais durante vários dias ou longos períodos. Em vista dessas dificuldades, o uso de dispositivos com maior capacidade de armazenamento e outras possibilidades de registros foi implementado para registrar o comportamento de pastejo em sistemas extensivos. Os colares GPS têm sido utilizados nos estudos comportamentais no campo da pecuária com o objetivo de registrar, além da localização, os comportamentos realizados, determinando o tipo e nível de atividade através de sistemas automatizados, sensores de movimento que registram os movimentos da cabeça ou acelerômetros para estimar atividades como pastejo. A informação obtida com essas ferramentas é valiosa para o estudo do comportamento dos bovinos de corte em pastagens, e serviria para entender a complexidade dos processos envolvidos na interação planta-animal-ambiente e o manejo de pastagem.

Palavras-chave: Etologia. GPS. Tecnologia de precisão. 


\section{Abstract}

The cattle grazing behaviour study can be considered an important tool to understand why animals choose some plants or graze moments. There is knowledge that animals possess skills that allow decision made during grazing process. Therefore, to study grazing behaviour, it is generally desirable observations to be made throughout the 24 hours of day. In food behaviour studies, in both wild and domestic ungulates, the use of precision technologies aims to facilitate the collection of information concerning animals and the environment by reducing the interference of the presence of the observers on animals' behaviour. However, observations of this type are not easy to make when they depend on the presence of human observers, due to a series of difficulties in data collection due to errors of registration or imprecision in the identification of the study area. This review aimed to identify some electronic devices as possible tools to evaluate the grazing behaviour of beef cattle in extensive systems. The first developed devices presented some limitations for their use in large areas or recording and storing behavioural information over several days or long periods. In view of these difficulties, the use of devices with greater storage capacity and other possibilities of records were implemented to record the grazing behaviour in extensive systems. The GPS collars were implemented in the behavioural studies in the field of livestock farming with the purpose of recording, in addition to location, the behaviours performed, determining the type and level of activity through automated systems, motion sensors that record movements of the head or accelerometers to record cattle speed estimating activities such as grazing. The information obtained with these tools is valuable for the study of the behaviour of beef cattle in grassland and would serve to understand the complexity of processes involved in plant-animal-environment interaction and pasture management.

Keywords: Ethology. GPS. Precision technology.

\section{Introdução}

Os estudos do comportamento de ingestão dos bovinos em pastejo geralmente analisam a interação planta-animal com a finalidade de responder perguntas relacionadas à nutrição animal, à ecofisiologia das pastagens e ao impacto sobre o desempenho animal. Assim, o comportamento de pastejo vem sendo amplamente estudado sob o ponto de vista nutricional (Forbes, 2007).

Alguns autores definem que o processo de pastejo poderia ocorrer em duas escalas temporais: uma de curto prazo, definida pela escala de minutos a horas de pastejo, e outra de longo prazo, definida pela escala de dias a semanas de pastejo (Laca e Demment, 1992; Bailey et al., 1996). Em função disso, para estudar o comportamento de pastejo, geralmente é desejável que as observações sejam feitas durante as 24 horas do dia (Carvalho et al., 2007). Observações deste tipo, porém, não são fáceis de serem feitas quando dependem da presença de observadores humanos, devido a uma série de dificuldades nas coletas de dados decorrentes de erros de registro, de imprecisão na identificação da área de estudo, além das limitações decorrentes do ambiente, como fatores físicos e climáticos, associados à fadiga dos observadores (Turner et al., 2000; Laca, 2009).

Para reduzir os riscos de ocorrência desses problemas e o efeito da presença dos humanos sobre o comportamento natural dos animais durante as observações, pode-se utilizar ferramentas tecnológicas como os dispositivos de registro eletrônico, que tornam possível avaliar o comportamento dos bovinos ao longo do tempo, com alto nível de detalhamento e em diferentes condições de criação (Adamczyk et al., 2013). É importante considerar, porém, que qualquer dispositivo colocado no animal pode interferir em parte no seu comportamento natural, pelo qual é necessário que o animal seja habituado previamente ao uso do dispositivo para que se tenha uma melhor apreciação do comportamento natural. Em outros contextos, estes dispositivos também poderiam auxiliar no estudo dos fatores individuais, como o temperamento, mas em situações que não se encontram frente à presença dos humanos, avaliando como esta característica poderia afetar, por exemplo, o comportamento de ingestão dos animais, assim como foi demostrado no estudo de Wesley et al. (2012).

Levando em conta estas considerações, o objetivo geral desta revisão foi identificar alguns dispositivos eletrônicos como possíveis ferramentas para avaliar o comportamento de pastejo de bovinos de corte em sistemas extensivos. 


\section{Generalidades sobre o comportamento de pastejo dos bovinos}

O entendimento do comportamento alimentar dos bovinos pode ser considerado uma ferramenta importante no manejo das pastagens, principalmente em sistemas extensivos. Existe ampla informação agronômica acerca da ecofisiologia das pastagens, além de numerosos estudos tentando entender por que os animais escolhem determinadas plantas ou momentos para pastejar (Provenza e Launchbaugh, 1999; Provenza et al., 2003). Os herbívoros ungulados possuem habilidades que permitem a tomada de decisões durante o processo de forrageamento, sendo capazes de memorizar os sítios, dentro de sua área de vida, onde encontrarão a biodiversidade das plantas preferencialmente ingeridas, otimizando a utilização de nutrientes e evitando aquelas plantas que poderiam conter toxinas (Provenza et al., 2003; Launchbaugh e Howery, 2005; Manteca et al., 2008). Desse modo, ao longo de sua história evolutiva, os ruminantes domésticos desenvolveram estratégias bem-sucedidas para aperfeiçoar seu comportamento de forrageamento, resultando no que foi definido como processo de pastejo (Carvalho et al., 1999).

Apesar disso, dada a complexidade dos processos envolvidos, alguns problemas de manejo das pastagens só podem ser bem compreendidos e resolvidos pela combinação de informações relacionadas aos animais e ao ambiente. Está descrito que várias dessas habilidades ou processos poderiam ser tanto herdados quanto aprendidos através da convivência com outros indivíduos do grupo (Launchbaugh et al., 1999). Ganskopp e Cruz (1999) determinaram a rapidez com que novilhos sem experiência expressam claramente suas preferências perante um novo conjunto de forragens quando são introduzidos junto a animais autóctones. Foi observado que os novilhos autóctones começaram imediatamente a pastejar as forragens preferidas, sendo que $90 \%$ de suas visitas foram realizadas em duas dessas gramíneas. Os novilhos sem experiência, por sua vez, começaram a expressar preferências alimentares similares às dos animais autóctones em menos de cinco minutos.

As teorias que tentam explicar a regulação do consumo de alimentos em ruminantes consideram mecanismos multifatoriais tanto físicos como fisiológicos do animal (Forbes, 2003, 2007). A regulação do consumo de alimento, portanto, é o resultado da interação entre informações de origem genética, estado fisiológico do animal e sinais das reservas intestinais e dos tecidos que são transmitidos através dos sistemas nervoso e endócrino (Mertens, 1996; Forbes, 2001). Entre os fatores bióticos e abióticos que regulam o consumo dos ruminantes, podemos considerar: (1) fatores bióticos - relacionados à qualidade nutricional da forragem (composição química e produtividade das plantas) (Bailey et al., 1996), ao comportamento social dos animais (Sibbald etal., 2000), aos efeitos das perturbações (possíveis ataques de predadores ou insetos), dentre outros (Howery et al., 1998; Broom, 2010); (2) fatores abióticos, tais como declividade do terreno, localização das fontes de água ou sal (Senft et al., 1987; Holechek, 1988; Smith, 1988; Ganskopp, 2001; Kaufmann et al., 2013), distância percorrida para obter alimentos, temperatura do ambiente, dentre outros (Riggs et al., 1990).

Os fatores supracitados podem ajudar a esclarecer por que áreas aparentemente pastejáveis muitas vezes não são exploradas pelos animais. Os bovinos, por exemplo, têm preferência por áreas mais planas e, por isso, geralmente não consomem a forragem presente em encostas com mais de $60 \%$ de inclinação, de maneira que as áreas com essas características não devem ser consideradas pastejáveis (Holechek, 1988). Já em relação às fontes de água, bovinos fazem pouco ou nenhum uso de áreas distantes $(>3,2 \mathrm{Km})$ das aguadas (Holechek, 1988). Em resumo, a seleção da dieta resultaria de sinais internos e externos percebidos pelo animal, determinando que a taxa do bocado seja adequada para obter o alimento que satisfaça as necessidades do mesmo (Gregorini et al., 2009; Villalba et al., 2009).

\section{Tecnologia de precisão auxiliando nos estudos do comportamento animal}

A utilização de tecnologias de precisão nos estudos do comportamento alimentar, tanto em ungulados selvagens quanto em domésticos, tem como objetivo facilitar a coleta de informações referentes aos animais e ao ambiente (Frost et al., 1997) e diminuir a interferência da presença dos 
observadores sobre o comportamento dos animais (Turner et al., 2000; Laca, 2009; Swain et al., 2011).

0 registro automático das atividades comportamentais dos animais pode ser realizado por meio de uma variedade de métodos que, no geral, permitem o registro objetivo dos dados. Como exemplo, podem ser citados: o sistema pneumático, que registra os movimentos da cabeça e as atividades de alimentação do animal com o propósito de estimar o tempo de ingestão (Duckworth e Shirlaw, 1955); equipamentos que foram adaptados, como o interruptor de mercúrio (O'Shea, 1969); ou ainda os vibracordes, que permitem estimar as atividades e, em especial, o tempo de pastejo de bovinos (Stobbs, 1970; Gwynne e Kingaby, 1976; Ruckebusch e Bueno, 1978).

Já com o objetivo de entender o comportamento de ingestão foram desenvolvidos sistemas que permitiam registrar os movimentos do maxilar, para determinar com exatidão o tempo despendido na mastigação (Black e Kenney, 1984, Penning et al., 1984; Matsui e Okubo, 1991), podendo, inclusive, ser registrado digitalmente (Rutter et al., 1997; Rutter, 2000; Ungar e Rutter, 2006), e interruptores de inclinação de mercúrio, tanto para ovelhas como para bovinos (Champion et al., 1997). Além desses dispositivos, foi desenvolvida também uma gama de pedômetros (Hart et al., 1993; Firk et al., 2002), sensores de aceleração piezoelétrica (Patterson et al., 1993; Bussmann et al., 1998a,b; Scheibe et al., 1998; Foerster et al., 1999), técnicas baseadas em campos magnéticos induzidos por transponder (Bollhalder e Messerli, 1997), em laser (Fehmi e Laca, 2001), software de controle de posição online automático (Schwarz et al., 2002), plataformas controladas por sensor (Van de Weerd et al., 2001) e análises automatizadas de vídeo (Noldus et al., 2001).

A maioria dos dispositivos mencionados acima apresentou algumas limitações para seu uso em grandes áreas ou para registrar e armazenar informações de comportamento durante vários dias ou períodos longos. Assim, surgiu a necessidade de colares autoajustáveis, que inicialmente foram projetados para uso em estudos sobre predação de ovelhas domésticas, onde os cordeiros foram monitorados desde o nascimento até um ano de idade. Foi observado que os mesmos não apresentaram problemas ao carregar os transmissores (Kolz e Johnson, 1980). Devido a isto, para estudar o comportamento em sistemas extensivos de pastejo, começou-se a utilizar dispositivos com maior capacidade de armazenamento, determinando, por exemplo, as distâncias percorridas por animal por dia, com a utilização de pedômetros (Rouda et al., 1990), e dispositivos de transmissão via radiofrequência para monitorar animais em confinamento durante vários dias (Sowell et al., 1998).

Para aumentar a precisão dos registros dos dados foram utilizados equipamentos com o sistema de posicionamento geográfico (GPS), que tornaram possível avaliar a distribuição espacial dos animais e analisar a dispersão com maior precisão (Moen et al., 1997; Rempel e Rodgers, 1997; Rutter et al., 1997; Hulbert et al., 1999; Turner et al., 2000). Estes dispositivos funcionam em conjunto com o sistema de informação geográfica (SIG), caracterizado por ser um conjunto de ferramentas úteis para a coleta, armazenamento, edição, processamento e apresentação de dados sobre os mapas ou sobre uma área específica (georreferenciada) (Turner et al., 2000; Burrough, 2001; Hulbert e French, 2001). Com este sistema é possível avaliar o comportamento de pastejo em ambientes heterogêneos, emergindo como uma importante ferramenta para compreensão do uso do espaço pelos bovinos em ecossistemas de pastagens (Parsons et al., 2001).

\section{Utilização de colares com GPS e sensores de movimento para avaliar o comportamento de pastejo}

Os colares com GPS foram utilizados inicialmente para estudar o comportamento de animais em vida selvagem (Moen et al., 1996; Dussault et al., 1999; Blake et al., 2001). Um dos primeiros estudos em que estes dispositivos foram utilizados para avaliar o comportamento de animais domésticos foi realizado com ovelhas. Estas foram rastreadas com o objetivo de correlacionar níveis de césio nas carcaças de animais que pastejaram áreas específicas (Roberts et al., 1995; Rutter et al., 1997). Estes dispositivos foram implementados nos 
estudos de comportamento de animais de produção com o propósito de registrar, além da localização, os comportamentos realizados, determinando o tipo e o nível de atividade por meio de sistemas automatizados para registro de atividades como sensores de movimentos do maxilar e da posição vertical do corpo (Rutter et al., 1997), movimentos gerais da cabeça, do pescoço ou do corpo (Relyea e Demarais, 1994), além de sensores que detectam os movimentos do eixo horizontal e vertical da cabeça ( $X$-act e $Y$-act, respectivamente) (Turner et al., 2000; Ganskopp, 2001). Entretanto, os dados coletados por esses sensores adicionais geralmente foram interpretados apenas considerando o animal ativo, representado por comportamento de alimentação e caminhada, ou inativo, representado como descansando (Müller e Schrader, 2003).

Assim, estes recursos tecnológicos passaram a ser utilizados para estudar o comportamento de bovinos em pastagens, fornecendo informações, por exemplo, para definir a seleção de áreas não contaminadas com placas de fezes (Dohi et al., 1991; Hutchings et al., 1998; Páscoa, 2005), para avaliar o uso de recursos como água (Pandey et al., 2009), minerais e suplementos alimentares que afetam a distribuição espacial dos animais na pastagem e, consequentemente, seu consumo (Ganskopp, 2001; Bailey et al., 2008; Valente et al., 2013), ou também para avaliar a biomassa de forragem e as diferenças no padrão de altura e de pastejo dos bovinos (Handcock et al., 2009). Dessa maneria, os colares GPS poderiam ser utilizados em estudos abordando a avaliação do bem-estar dos animais ou em estudos sobre o uso de pastagens (Müller e Schrader, 2003), onde é reconhecida a dificuldade de se obter dados apropriados com a presença de humanos fazendo os registros visualmente.

No estudo realizado por Ganskopp (2001) foi avaliada a eficácia da manipulação do sal e da água na distribuição dos bovinos em grandes pastagens, e para monitorar o deslocamento e as atividades dos animais foram utilizados colares GPS. Com base nos resultados obtidos, os autores concluíram que a água é a ferramenta mais eficiente para promover alterações na distribuição dos animais na pastagem. Pandey et al. (2009) quantificaram o tempo que bovinos com mais de 3 anos de idade permaneciam perto ou em locais de água nas diferentes estações do ano em uma fazenda de produção de bezerros no sul da Flórida e, para isso, utilizaram em média dois a quatro colares GPS por parcela. Esses autores observaram maior presença de animais perto de locais com água durante os períodos mais quentes em comparação aos períodos frios $(11,45 \pm 0,39 \%$ versus $6,09 \pm 0,69 \%$ ).

As informações fornecidas por esses dispositivos (colar GPS com ou sem sensores de movimentos ou com acelerômetros, entre outros) podem ser usadas para prever ou agrupar as atividades dos animais em um período de 24 horas por vários dias ou meses (por exemplo, tempo gasto em pastoreio, repouso, etc), conseguindo estimativas confiáveis dos custos de energia das atividades de bovinos em pastagem (Brosh et al., 2006). Para isso, há diferentes algoritmos e modelos matemáticos que poderiam predizer os comportamentos dos animais, como, por exemplo, modelos que utilizam dados de GPS submétricos (Schlecht et al., 2004), modelos de regressão e análise discriminante (Ungar et al., 2005; Barbari et al., 2006), e análise de cluster (K-means) (Schwager et al., 2007).

Outras ferramentas estatísticas, como as árvores de decisão e classificação (De'ath e Fabricius, 2000), foram utilizadas por vários autores para converter os dados eletrônicos provenientes dos sensores de movimentos em comportamentos categóricos (Nadimi et al., 2008). Estes algoritmos de árvores de decisão mostraram alta precisão e especificidade para estimar comportamentos de estado como o pastejo e o nível de atividade a partir dos dados do GPS (Nadimi et al., 2008; Robert et al., 2009), dos sensores de inclinação (Ungar et al., 2005, 2011; Umstätter et al., 2008; Augustine e Derner, 2013) ou dos colares com acelerômetros (Swain et al., 2008; González et al., 2015). Este último dispositivo já havia sido apontado como uma ferramenta útil para a coleta de dados confiáveis sobre o padrão temporal de comportamento geral e níveis de atividade sem restringir a liberdade de movimento do animal. 0 colar GPS com sensores de inclinação é um desses dispositivos que pode auxiliar na avaliação do comportamento de pastejo dos bovinos que, por meio do método de árvores de decisões, consegue obter os limiares para identificar três comportamentos básicos considerados estados (pastejo, ruminação e não pastejo), obtendo maior 
precisão e especificidade para a categoria pastejo (Aguilar, 2016). Lomba et al. (2015) demonstraram que seria possível classificar os comportamentos primários utilizando algoritmos de árvores de decisão. Os autores, porém, sugerem que seria importante incorporar outras variáveis ambientais para identificar comportamentos secundários não identificados inicialmente. Assim, Lomba et al. (2017) desenvolveram um software para automatizar a rotulação dos dados, aperfeiçoando o trabalho de observação, importação e manipulação dos dados obtidos em campo. A ferramenta, além de reduzir o tempo de rotulação dos dados, também elimina a possibilidade de erros na transcrição das anotações manuais para o arquivo digital, melhorando a acurácia de classificações para 93,2\%.

\section{Limitações dos colares GPS}

Alguns autores, como Rothwell et al. (2011) e Adams et al. (2013), demostraram limitações dos colares GPS quando utilizados em áreas pequenas, uma vez que as distâncias calculadas a partir do GPS foram imprecisas. Também foi observado que elementos como coberturas de árvores e cercas elétricas podem produzir algum erro adicional e interferir na precisão dos pontos georreferenciados (Agouridis et al., 2004; Ganskopp e Jonhson, 2007).

Assim como foi citado por Adams et al. (2013), no geral o receptor GPS está sujeito a dois tipos de erros: 1) receptores podem não adquirir informações durante um horário pré-definido (dados perdidos, taxa de correção de sucesso); 2) locais sinalizados podem ser espacialmente imprecisos, referido como erro de localização. Esses dois tipos de erro são influenciados por inúmeros fatores ambientais (topografia, características da vegetação, particularmente aquelas associadas à composição de espécies, complexidade estrutural, entre outros) (Frair et al., 2010) e tecnológicos, que podem afetar a transmissão do sinal dos satélites para os receptores (número de satélites presentes e sua configuração geométrica) (Moen et al., 1996; Di Orio et al., 2003). As correções posicionais são influenciadas pelo número de satélites que intervêm na triangulação, porém, uma correção posicional tridimensional (3-D) é obtida quando os sinais de quatro ou mais satélites são usados, e uma posição bidimensional (2-D) é adquirida só com três satélites (Rempel et al., 1995; Di Orio et al., 2003). Também é importante conhecer a configuração geométrica dos satélites disponíveis, conhecida como diluição da precisão (DOP; por exemplo, horizontal-HDOP ou vertical-VDOP) (Adams et al., 2013). Valores baixos de DOP são obtidos quando os satélites são espaçados, resultando em erros de triangulação menores e correções posicionais mais precisas do que correções associadas a valores altos de DOP (representando uma geometria pobre de satélite) (Rempel et al., 1995; Moen et al., 1997). Outros fatores técnicos que influenciam a perda de dados e a falha na precisão da localização incluem o enfraquecimento da bateria do GPS (Gau et al., 2004), diferenças nas marcas dos colares, mau funcionamento eletrônico e erros em relógios de satélite (Adams et al., 2013).

Outro ponto importante a ser considerado é que o tempo de uso do colar é condicionado pelo intervalo de coleta dos dados. Sabe-se que intervalos mais curtos (a cada 10 segundos ou menos, por exemplo) promovem maior gasto de bateria, mas, como foi demonstrado por Swain et al. (2008), um intervalo de gravação a cada 10 segundos em áreas de $100 \mathrm{~m}^{2}$ reduziria para $1 \%$ o erro de previsão da velocidade calculada. Dessa forma, nos estudos em que são necessários intervalos de registro curtos, o tempo de uso do colar entre as recargas da bateria será menor, implicando em maior necessidade de manejo dos animais para colocação e retirada dos colares GPS.

Para solucionar esses inconvenientes é possível utilizar colares GPS com descarregamento remoto. Nesse sentido, González et al. (2015) propuseram uma metodologia não supervisionada para tratamento de dados de alta resolução espacial e temporal, gerados a partir de acelerômetros triaxiais ( 4 e $10 \mathrm{~Hz}$ ), montados em colares equipados com GPS, com monitoramento automático e em tempo real do comportamento de bovinos. A implementação de rastreamento em tempo real e o descarregamento remoto dos dados permitiu aos pesquisadores ter uma melhor aproximação da distribuição dos animais e das respostas a estímulos agudos e de curto prazo em relação aos padrões comportamentais de longo prazo, proporcionando 
economia de tempo e custo aos pesquisadores e gerentes de recursos naturais na tomada de decisões (Clark et al., 2006).

Existem dispositivos, colares com GPS e sensores que vêm de fábrica com a programação fechada, não sendo possível, por exemplo, modificar os intervalos de coleta ou programar para poupar bateria. Também existem GPS que podem ser programados de acordo com os objetivos da pesquisa que se pretende realizar, porém, geralmente custam mais caro. Para o estudo do comportamento animal, portanto, é importante considerar que, dependendo do intervalo de tempo entre registros, os colares são eficientes para registrar apenas comportamentos de longa duração (em casos de intervalo de registro longo) ou de longa e curta duração (em casos de intervalo de registro curto).

Moen et al. (1996), usando colares GPS com programação fechada e intervalos de 5 minutos em alces, descobriram que um animal poderia sair e retornar ao mesmo ponto no intervalo de 5 minutos, fazendo com que uma categoria comportamental (por exemplo, beber água) fosse subestimada. Ganskopp (2001) e Ungar et al. (2005) também demonstraram que o consumo de água e sal foram eventos de curta duração e pouco frequentes, sendo mais difíceis de serem identificados separadamente das demais categorias comportamentais. Assim, considera-se que a programação do fabricante em intervalos de 5 minutos foi razoável para detectar comportamentos do tipo estado, como pastejo e ruminação, porém não foi boa para detectar comportamentos eventuais.

Resultados semelhantes foram demonstrados por Turner et al. (2000) e Ungar et al. (2005), que usaram os mesmos intervalos de 5 minutos e relataram alta acurácia para classificar as atividades de pastejo e repouso. Nesse sentido, para que a interpretação dos dados coletados seja feita de forma correta, é indispensável que os usuários procurem assistência técnica ou de pesquisadores que possam instruí-los sobre o uso da ferramenta e que a mesma esteja de acordo com as necessidades e os objetivos da utilização no campo.

Além disso, em muitas ocasiões as empresas não fornecem as fórmulas com que realizam os cálculos internos das variáveis prontas fornecidas pelo colar e, por esta razão, é recomendado sempre realizar uma validação do colar nas condições de campo, onde serão realizadas as coletas dos dados. Lomba et al. (2015) demonstraram que ainda existem problemas relacionados à influência da presença do observador no habitat do animal para poder aferir o que o colar GPS coleta e as limitações do homem, como a fadiga em trabalhos que exigem longos períodos de observação. Em geral, os pesquisadores ou usuários realizaram esse processo de observação utilizando caneta e papel (Nadimi et al., 2008; Zheng et al., 2008; Godsk e Kjaergaard, 2011), sendo um processo trabalhoso e que pode gerar erros, por exemplo, na anotação do tempo ou no momento de transferir os dados do papel para o formato digital.

Embora estes dispositivos ainda não sejam suficientemente econômicos para serem utilizados em grande escala, poderiam fornecer informações interessantes e precisas das atividades dos animais nas pastagens. No entanto, a complexidade do processamento e entendimento das informações fornecidas por estes dispositivos ainda pode ser considerada uma limitante, devido à sofisticação dos métodos para análise dos dados e à grande quantidade de informações, que permitem apenas uma baixa resolução temporal ou que são aplicáveis apenas a uma área espacial restrita (Müller e Schrader, 2003). Isso mostra que, apesar dos grandes avanços no estudo do comportamento alimentar desde sua implementação, ainda há desafios a serem superados.

\section{Utilização de colares GPS para estudos com enfoque em outros comportamentos}

Embora existam informações sobre a aplicação dessa tecnologia para estudos do comportamento alimentar de bovinos em pastagens, como descrito por Swain et al. (2011) e Anderson et al. (2013), ainda é pouco explorada a utilização da tecnologia de precisão para entender algumas características individuais dos bovinos em outros contextos ou situações. 0 estudo de Wesley et al. (2012) com vacas mostrou que existem diferenças individuais nos animais em relação à velocidade de consumo e à distância percorrida, quando os mesmos são mantidos em confinamento versus em pastagens, 
considerando que alguma característica do indivíduo (síndrome comportamental) poderia estar influenciando essas respostas.

MacKay et al. (2013), utilizando acelerômetros, avaliaram os níveis de atividade individual dos animais e a relação que poderia existir entre testes de temperamento (flight speed e chute score) e escores de comportamento social (agressividade e deslocamento no cocho). Estes autores concluíram que animais mais reativos foram mais ativos nos currais do confinamento comparados àqueles mais tranquilos. Além disso, os animais que deslocavam outros novilhos no cocho eram os que permaneciam mais tempo em pé e deitavam por menos tempo. Este foi um dos primeiros estudos que utilizou dispositivos eletrônicos e foi capaz de demonstrar que os testes de temperamento a curto prazo estão relacionados ao comportamento de longo prazo.

Em um estudo realizado com búfalas leiteiras, no qual foi medida a distância média percorrida diariamente (calculada com base nas informações obtidas por meio de colares GPS), não foram encontradas correlações significativas entre essa variável e a reatividade das fêmeas durante a ordenha, demonstrando que, provavelmente, estas características sejam independentes (Carvalhal et al., 2017). Nesse mesmo estudo, também não foi encontrada relação entre a distância percorrida e a produção de leite e os teores de gordura e proteína. De maneira semelhante, Lathrop et al. (1988) observaram que os padrões de uso da pastagem não influenciaram a produção de leite.

Já Wesley et al. (2012) relataram que os animais que se deslocaram por uma distância maior tiveram maior ganho de peso, tendiam a ter bezerros mais pesados e período de anestro mais curto. Esses resultados contrastam com a ideia de que um maior deslocamento causa maior gasto de energia e, consequentemente, menor produção do animal. O comportamento exploratório também pode estar relacionado às condições de saúde dos animais. Gustafson (1993) observou que vacas leiteiras que se deslocaram 0,5 - $3 \mathrm{~km} /$ dia tiveram melhor saúde quando comparadas às vacas mantidas em ambiente restrito, resultando em menor frequência de tratamentos para doenças e menos casos de mastite subclínica.

\section{Conclusão}

O comportamento de pastejo em bovinos é influenciado por diversos fatores relacionados ao animal e ao ambiente, como origem genética, estado fisiológico e comportamento social dos animais, qualidade nutricional da forragem, declividade do terreno, localização das fontes de água e suplementação, temperatura ambiente, entre outros. Devido à complexidade da interação entre esses fatores e às dificuldades na obtenção de informações referentes a alguns deles, a utilização de tecnologias de precisão contribui facilitando a coleta de informações relacionadas aos animais e ao ambiente.

Embora existam algumas limitações no uso dessas tecnologias, as informações que podem ser obtidas com essas ferramentas são valiosas para o estudo do comportamento de bovinos de corte em pastagens, e serviriam para entender a complexidade dos processos envolvidos na interação planta-animal-ambiente. Assim, é necessário que mais pesquisas científicas sejam realizadas com o objetivo de avaliar novas formas de utilização desses equipamentos e quais outras informações podem ser extraídas a partir deles, de maneira a contribuir com o entendimento do comportamento de pastejo, bem como estudos que proponham novos métodos de processamento das informações obtidas, facilitando a utilização prática desse tipo de tecnologia.

\section{Referências}

Adamczyk K, Pokorska J, Makulska J, Earley B, Mazurek M. Genetic analysis and evaluation of behavioural traits in cattle. Livest Sci. 2013;154(1-3):1-12.

Adams AL, Dickinson KJM, Robertson BC, Van Heezik Y. An Evaluation of the Accuracy and Performance of Lightweight GPS Collars in a Suburban Environment. PLoS ONE. 2013;8(7):e68496.

Agouridis CT, Stombaugh TS, Workman SR, Koostra BK, Edwards DR, Vanzant ES. Suitability of a GPS collar for grazing studies. Trans ASAE. 2004;47(4):1321-9. 
Aguilar NMA. Avaliação dos comportamentos de pastejo e suas relações com caracteres individuais dos bovinos de corte [tese]. Jaboticabal: Universidade Estadual Paulista; 2016.

Anderson DM, Estell RE, Cibils AF. Spatiotemporal cattle data - a plea for protocol standardization. Positioning. 2013;4(1):115-36.

Augustine DJ, Derner JD. Assessing herbivore foraging behavior with GPS collars in a semiarid grassland. Sensors (Basel). 2013;13(3):3711-23.

Bailey DW, Gross JE, Laca EA, Rittenhouse LR, Coughenour MB, Swift DM, et al. Mechanisms that result in large herbivore grazing distribution patterns. J Range Manage. 1996;49(5):386-400.

Bailey DW, Vanwagoner HC, Weinmeister R, Jensen D. Comparison of low-moisture blocks and salt for manipulating grazing patterns of beef cows. J Anim Sci. 2008;86(5):1271-7.

Barbari M, Conti L, Koostra BK, Masi G, Sorbetti Guerri F, Workman SR. The use of global positioning and geographical information systems in the management of extensive cattle grazing. Biosyst Eng. 2006;95(2):271-80.

Black JL, Kenney PA. Factors affecting diet selection by sheep. II. Height and density of Pasture. Aust J Agric Res. 1984;35(4):565-78.

Blake S, Hamilton ID, Karesh WB. GPS Telemetry of forest elephants in central Africa: results of a preliminary study. Afr J Ecol. 2001;39(2):178-86.

Bollhalder H, Messerli HK. Ein Tierortungssystem zur automatischen Erfassung des Aufenthaltsortes und der Aktivität von Kühen im Laufhof und im Laufstall. Agrartech Forsch-Agr. 1997;3(1):2-10.

Broom DM. Cognitive ability and awareness in domestic animals and decisions about obligations to animals. Appl Anim Behav Sci. 2010;126(1-2):1-11.

Brosh A, Henkin Z, Ungar ED, Dolev A, Orlov A, Yehuda Y, et al. Energy cost of cows' grazing activity: Use of the heart rate method and the Global Positioning System for direct field estimation. J Anim Sci. 2006;84(7):1951-67.
Burrough P. GIS and geostatistics: Essential partners for spatial analysis. Environ Ecol Stat. 2001;8(4):361-77.

Bussmann JBJ, van de Laar YM, Neeleman MP, Stam HJ. Ambulatory accelerometry to quantify motor behaviour in patients after failed back surgery: a validation study. Pain. 1998a;74(2-3):153-61.

Bussmann JBJ, Tulen JHM, van Herel ECG, Stam HJ. Quantification of physical activities by means of ambulatory accelerometry: a validation study. Psychophysiology. 1998b;35(5):488-96.

Carvalhal MVL, Sant'Anna AC, Páscoa AG, Jung J, Costa MJRP. The relationship between water buffalo cow temperament and milk yield and quality traits. Livest Sci. 2017;198:109-14.

Carvalho PCF, Prache S, Damasceno JC. O processo de pastejo: desafios da procura e apreensão da forragem pelo herbívoro. $36^{\circ}$ Reunião Anual da Sociedade Brasileira de Zootecnia; 1999; Porto Alegre, RS. Porto Alegre: Sociedade Brasileira de Zootecnia; 1999. p. 253-68.

Carvalho PCF, Kozloski GV, Ribeiro Filho HMN, Reffatti MV, Genro TCM, Euclides VPB. Avanços metodológicos na determinação do consumo de ruminantes em pastejo. $\mathrm{R}$ Bras Zootec. 2007;36(supl esp):151-70.

Champion RA, Rutter SM, Penning PD. An automatic system to monitor lying, standing and walking behaviour of grazing animals. Appl Anim Behav Sci. 1997;54(4):291-305.

Clark PE, Johnson DE, Kniep MA, Jermann P, Huttash B, Wood A, et al. An advanced, low-cost, GPS-based animal tracking system. Rangeland Ecol Manage. 2006;59(3):334-40.

De'ath G, Fabricius KE. Classification and regression trees: a powerful yet simple technique for ecological data analysis. Ecology. 2000;81(11):3178-92.

Di Orio AP, Callas R, Schaefer RJ. Performance of two GPS telemetry collars under different habitat conditions. Wildl Soc Bull. 2003;31(2):372-9.

DohiH,YamadaA,EntsuS.Cattlefeedingdeterrentsemitted from cattle feces. J Chem Ecol. 1991;17(6):1197-203. 
Duckworth JE, Shirlaw DW. The development of an apparatus to record the jaw movements of cattle. Br J Anim Behav. 1955;3(2):56-60.

Dussault C, Courtois R, Ouellet JP, Huot J. Evaluation of GPS telemetry collar performance for habitat studies in the boreal forest. Wildl Soc Bull. 1999;27(4):965-72.

Fehmi JS, Laca EA. A note on using a laser-based technique for recording of behaviour and location of free-ranging animals. Appl Anim Behav Sci. 2001;71:335-9.

Firk R, Stamer E, Junge W, Krieter J. Automation of oestrus detection in dairy cows: a review. Livest Prod Sci. 2002;75(3):219-32.

Foerster F, Smeja M, Fahrenberg J. Detection of posture and motion by accelerometry: a validation study in ambulatory monitoring. Comput Human Behav. 1999;15(5):571-83.

Forbes JM. Consequences of feeding for future feeding. Comp Biochem Physiol A Mol Integr Physiol. 2001;128(3):461-8.

Forbes JM. The multifactorial nature of food intake control. J Anim Sci. 2003;81(14 supl 2):E139-44.

Forbes JM. A personal view of how ruminant animals control their intake and choice of food: minimal total discomfort. Nutr Res Rev. 2007;20(2):132-46.

Frair JL, Fieberg J, Hebblewhite M, Cagnacci F, DeCesare NJ, Pedrotti L. Resolving issues of imprecise and habitat-biased locations in ecological analyses using GPS telemetry data. Philos Trans R Soc Lond B Biol Sci. 2010;365(1550):2187-200.

Frost AR, Schofield CP, Beaulah SA, Mottram TT, Lines JA, Wathes CM. A review of livestock monitoring and the need for integrated systems. Comput Electron Agric. 1997;17(2):139-59.

Ganskopp D, Cruz R. Selective differences between naive and experienced cattle foraging among eight grasses. Appl Anim Behav Sci. 1999;62(4):293-303.

Ganskopp D. Manipulating cattle distribution with salt and water in large arid-land pastures: a GPS/GIS assessment. Appl Anim Behav Sci. 2001;73(4):251-62.

Ganskopp D, Johnson DD. GPS error in studies addressing animal movements and activities. Rangeland Ecol Manage. 2007;60:350-8.

Gau RJ, Mulders R, Ciarniello LJ, Heard DC, Chetkiewicz CLB, et al. Uncontrolled field performance of Televilt GPS-SimplexTM collars on grizzly bears in western and northern Canada. Wildl Soc Bull. 2004;32:693-701.

González LA, Bishop-Hurley GJ, Handcock RN, Crossman C. Behavioral classification of data from collars containing motion sensors in grazing cattle. Comput Electron Agric. 2015;110:91-102.

Godsk T, Kjærgaard MB. High classification rates for continuous cow activity recognition using low-cost GPS positioning sensors and standard machine learning techniques. In: Perner P (E.). Advances in Data Mining. Applications and Theoretical Aspects. Berlin: Springer; 2011. p. 174-88.

Gregorini P, Gunter SA, Beck PA, Caldwell J, Bowman MT, Coblentz WK. Short-term foraging dynamics of cattle grazing swards with different canopy structures. J Anim Sci. 2009;87(11):3817-24

Gustafson GM. Effects of daily exercise on the health of tied dairy cows. Prev Vet Med. 1993;17(3-4):209-23.

Gwynne MD, Kingaby GP. A grazing recorder harness for use with Zebu type cattle. J Range Manage. 1976;29(3):260-2.

Handcock RN, Swain DL, Bishop-Hurley GJ, Patison KP, Wark T, Valencia P, Corke P, O’Neill CJ. Monitoring animal behaviour and environmental interactions using wireless sensor networks, GPS collars and satellite remote sensing. Sensors (Basel). 2009;9(5):3586-603.

Hart RH, Bissio J, Samuel MJ, Waggoner JW. Grazing systems, pasture size, and cattle grazing behavior, distribution and gains. J Range Manage. 1993;46(1):81-7.

Holechek JL. An approach for setting the stocking rate. Rangelands. 1988;10(1):10-4. 
Howery LD, Provenza FD, Banner RE, Scott CB. Social and environmental factors influence cattle distribution on rangeland. Appl Anim Behav Sci. 1998;55(3-4):231-44.

Hulbert IAR, Waterhouse A, Gordon P, Morgan-Davies C. Silvi-pastoralism for the uplands of Scotland. A new approach to an old problem - the integration of farming and forestry. Scott For. 1999;53(4):231-5.

Hulbert IAR, French J. The accuracy of GPS for wildlife telemetry and habitat mapping. J Appl Ecol. 2001;38(4):869-78.

Hutchings MR, Kyriazakis I, Anderson DH, Gordon IJ, Coop RL. Behavioural strategies used by parasitised and nonparasitised sheep to avoid ingestion of gastrointestinal nematodes. Anim Sci. 1998;67(1):97-106.

Kaufmann J, Bork EW, Blenis PV, Alexander MJ. Cattle habitat selection and associated habitat characteristics under free-range grazing within heterogeneous Montane rangelands of Alberta. Appl Anim Behav Sci. 2013;146(1-4):1-10.

Kolz AL, Johnson RE. Self-Adjusting Collars for Wild Mammals Equipped with Transmitters. J Wildl Manag. 1980;44(1):273-5.

Laca EA, Demment MW. Modelling intake of a grazing ruminant in a heterogeneous environment. 24th International Symposium on Vegetation: Herbivore Relationships; 1992; Nova York. Nova York: Academic Press; 1992. p. 57-76.

Laca EA. Precision livestock production: tools and concepts. R Bras Zootec. 2009;38(no spe):123-32.

Lathrop WJ, Kress DD, Havstad KM, Doornbos DE, Ayers EL. Grazing behavior of rangeland beef cows differing in milk production. Appl Anim Behav Sci. 1988;21(4):315-27.

Launchbaugh KL, Walker JW, Taylor CA. Foraging behavior: experience or inheritance? In: Launchbaugh KL, Mosley JC, Sanders KD (EE.). Grazing Behavior of Livestock and Wildlife - Pacific Northwest Range Short Course. Moscow, ID: University of Idaho; 1999. p. 28-35.

Launchbaugh KL, Howery LD. Understanding landscape use patterns of livestock as a consequence of foraging behavior. Rangeland Ecol Manage. 2005;58(2):99-108.

Lomba LFD, Jesus L, Rubinsztejn HKS, Gonda L, Pires PP. 0 uso de inteligência artificial na identificação do comportamento bovino. In: Anais eletrônicos do X Congresso Brasileiro de Agroinformática. Ponta Grossa: SBIAGRO; 2015.

Lomba LFD, Jesus L, Rubinsztejn HKS, Gonda L, Pires PP. Sistema para Monitoramento da Movimentação Bovina e Aferição dos Comportamentos. In: Anais do XI Congresso Brasileiro de Agroinformática. Campinas: Universidade Estadual de Campinas; 2017. p. 363-373.

MacKay JRD, Turner SP, Hyslop J, Deag JM, Haskell MJ. Short-term temperament tests in beef cattle relate to long-term measures of behavior recorded in the home pen. J Anim Sci. 2013;91(10):4917-24.

Manteca X, Villalba JJ, Atwood SB, Dziba L, Provenza FD. Is dietary choice important to animal welfare? J Vet Behav. 2008;3(5):229-39.

Matsui K, Okubo T. A method for quantification of jaw movements suitable for use on free-ranging cattle. Appl Anim Behav Sci. 1991;32(1-2):107-16.

Mertens DR. Methods in modelling feeding behaviour and intake in herbivores. Ann Zootech. 1996;45(supl 1):153-64.

Moen R, Pastor J, Cohen Y, Schwartz CC. Effects of moose movement and habitat use on GPS collar performance. J Wildl Manage. 1996;60(3):659-68.

Moen R, Pastor J, Cohen Y. Accuracy of GPS telemetry collar locations with differential correction. J Wildl Manage. 1997;61(2):530-9.

Müller R, Schrader L. A new method to measure behavioural activity levels in dairy cows. Appl Anim Behav Sci. 2003;83(4):247-58.

Nadimi ES, Søgaard HT, Bak T. ZigBee-based wireless sensor networks for classifying the behaviour of a herd of animals using classification trees. Biosyst Eng. 2008;100(2):167-76. 
Noldus LP, Spink AJ, Tegelenbosch RA. EthoVision: a versatile video tracking system for automation of behavioral experiments. Behav Res Methods Instrum Comput. 2001;33(3):398-414.

O'Shea J. Evaluation of a simple device for measuring the time animals spend grazing. Irish J Agr Food Res. 1969;8(3):329-35.

Pandey V, Kiker GA, Campbell KL, Williams MJ, Coleman SW. GPS Monitoring of Cattle Location Near Water Features in South Florida. Appl Eng Agric. 2009;25(4):551-62.

Parsons AJ, Schwinning S, Carrère P. Plant growth functions and possible spatial and temporal scaling errors in models of herbivory. Grass Forage Sci. 2001;56(1):21-34.

Páscoa AG. Comportamento de bovinos da raça Nelore mantidos em pastagem de Cynodon spp cv Tifton 85: defecação e rejeição da forragem contaminada por fezes [dissertação]. Jaboticabal: Universidade Estadual Paulista; 2005. 50 p.

Patterson SM, Krantz DS, Montgomery LC, Deuster PA, Hedges SM, Nebel LE. Automated physical activity monitoring: validation and comparison with physiological and self-report measures. Psychophysiology. 1993;30(3): 296-305.

Penning PD, Steel GL, Johnson RH. Further development and use of an automatic recording system in sheep grazing studies. Grass Forage Sci. 1984;39(4):345-51.

Provenza FD, Launchbaugh KL. Foraging on the Edge of Chaos. In: Launchbaugh KL, Mosley JC, Sanders KD (EE.). Grazing Behavior of Livestock and Wildlife. Moscow, ID: University of Idaho; 1999. p. 1-12.

Provenza FD, Villalba JJ, Dziba LE, Atwood SB, Banner RE. Linking herbivore experience, varied diets, and plant biochemical diversity. Small Rumin Res. 2003;49(3):257-74.

Relyea RA, Demarais S. Activity of Desert Mule Deer during the Breeding Season. J Mammal. 1994;75(4):940-9.

Rempel RS, Rodgers AR, Abraham KF. Performance of a
GPS animal location system under boreal forest canopy. J Wildl Manage. 1995;59(3):543-51.

Rempel RS, Rodgers AR. Effects of differential correction on accuracy of a GPS animal location system. J Wildl Manage. 1997;61(2):525-30.

Riggs R, Urness PJ, Gonzalez KA. Effects of domestic goats on deer wintering in Utah oakbrush. J Range Manage. 1990;43(3):229-34.

Robert B, White BJ, Renter DG, Larson RL. Evaluation of three-dimensional accelerometers to monitor and classify behavior patterns in cattle. Comput Electron Agric. 2009;67(1-2):80-4.

Roberts G, Williams A, Last JD, Penning PD, Rutter SM. A low-power postprocessed DGPS system for logging the locations of sheep on hill pastures. J Inst Navigation. 1995;42(2):327-36.

Rothwell ES, Bercovitch FB, Andrews JRM, Anderson MJ. Estimating daily walking distance of captive African elephants using an accelerometer. Zoo Biol. 2011;30(5):579-91.

Rouda RR, Anderson DM, Murray LW, Smith JN. Distance traveled by free-ranging supplemented and nonsupplemented lactating and non-lactating cows. Appl Anim Behav Sci. 1990;28(3):221-32.

Ruckebusch Y, Bueno L. An analysis of ingestive behaviour and activity of cattle under field conditions. Appl Anim Ethol. 1978;4(4):301-13.

Rutter SM, Champion RA, Penning PD. An automatic system to record foraging behaviour in free-ranging ruminants. Appl Anim Behav Sci. 1997;54(2-3):185-95.

Rutter SM. Graze: A program to analyze recordings of the jaw movements of ruminants. Behav Res Methods Instrum Comput. 2000;32(1):86-92.

Scheibe KM, Schleusner T, Berger A, Eichhorn K, Langbein J, Dal Zotto L, et al. ETHOSYS (R) - new system for recording and analysis of behaviour of free-ranging domestic animals and wildlife. Appl Anim Behav Sci. 1998;55(3-4):195-211. 
Schlecht E, Hülsebusch C, Mahler F, Becker K. The use of differentially corrected global positioning system to monitor activities of cattle at pasture. Appl Anim Behav Sci. 2004;85(3-4):185-202.

Schwager M, Anderson DM, Butler Z, Rus D. Robust classification of animal tracking data. Comput Electron Agric. 2007;56(1):46-59.

Schwarz S, Hofmann MH, Gutzen C, Schlax S, von der Emde G. VIEWER: a program for visualising, recording, and analysing animal behaviour. Comput Methods Programs Biomed. 2002;67(1):55-66.

Senft RL, Coughenour MB, Bailey DW, Rittenhouse LR, Sala OE, Swift DM. Large herbivore foraging and ecological hierarchies. BioScience. 1987;37(11):789-99.

Sibbald AM, Smith DJF, Hooper RJ. Individual differences in sociability and their consequences for foraging in sheep. Proceedings of Annual Meeting the British Society of Animal Science; 2000. p. 129.

Smith MS. Modelling: three approaches to predicting how herbivore impact is distributed in rangelands. Las Cruces: New Mexico State University Library; 1988. 628 p.

Sowell BF, Bowman JGP, Branine ME, Hubbert ME. Radio frequency technology to measure feeding behavior and health of feedlot steers. Appl Anim Behav Sci. 1998;59(4):277-84.

Stobbs TH. Automatic measurement of grazing time by dairy cows on tropical grass and legume pastures. Trop Grassl. 1970;4(4):237-44.

Swain DL, Wark T, Bishop-Hurley GJ. Using high fix rate GPS data to determine the relationships between fix rate, prediction errors and patch selection. Ecol Modell. 2008;212(3-4):273-9.

Swain DL, Friend MA, Bishop-Hurley GJ, Handcock RN, WarkT.Trackinglivestockusingglobal positioningsystems - are we still lost? Anim Prod Sci. 2011;51(3):167-75.

Turner LW, Udal MC, Larson BT, Shearer SA. Monitoring cattle behavior and pasture use with GPS and GIS. Can J Anim Sci. 2000;80(3):405-13.
Umstätter C, Waterhouse A, Holland JP. An automated sensor-based method of simple behavioural classification of sheep in extensive systems. Comput Electron Agric. 2008;64(1):19-26.

Ungar ED, Henkin Z, Gutman M, Dolev A, Genizi A, Ganskopp D. Inference of animal activity from GPS collar data on free-ranging cattle. Rangeland Ecol Manage. 2005;58(3):256-66.

Ungar ED, Rutter SM. Classifying cattle jaw movements: comparing IGER behaviour recorder and acoustic techniques. Appl Anim Behav Sci. 2006;98(1-2):11-27.

Ungar ED, Schoenbaum I, Henkin Z, Dolev A, Yehuda $\mathrm{Y}$, Brosh A. Inference of the activity timeline of cattle foraging on a Mediterranean woodland using GPS and pedometry. Sensors (Basel). 2011;11(1):362-83.

Valente EEL, Paulino MF, Detmann E, Valadares Filho SC, Chizzotti ML, Silva AG, et al. Grazing Behavior and Locomotion of Young Bulls Receiving Different Nutritional Plans in a Tropical Pasture. Asian-Australas J Anim Sci. 2013;26(12):1717-25.

Van de Weerd HA, Bulthuis RJ, Bergman AF, Schlingmann F, Tolboom J, Van Loo PL, et al. Validation of a new system for the automatic registration of behaviour in mice and rats. Behav Processes. 2001;53(1-2):11-20.

Villalba JJ, Soder KJ, Laca EA. Understanding diet selection in temperate biodiverse pasture systems. Rangeland Ecol Manage. 2009;62(5):387-8.

Wesley RL, Cibils AF, Mulliniks JT, Pollak ER, Petersen MK, Fredricksond EDL. An assessment of behavioural syndromes in rangeland-raised beef cattle. Appl Anim Behav Sci. 2012;139(3-4):183-94.

Zheng Y, Liu L, Wang L, Xie X. Learning transportation mode from raw gps data for geographic applications on the web. Proceedings of the 17th International World Wide Web Conference (WWW2008). Beijing, China; 2008. p. 247-56. 